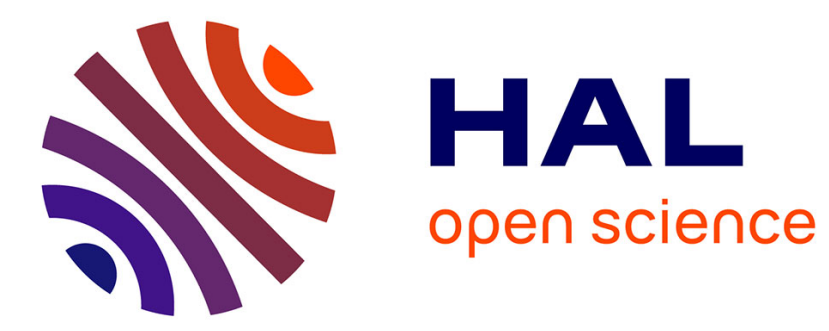

\title{
Dynamique lacustre Holocène dans la sebkha al Kalbiyya (Tunisie centre orientale)
}

Abdelkarim Boujelben, Vincent Lebreton, Mohamed Raouf Karray

\section{To cite this version:}

Abdelkarim Boujelben, Vincent Lebreton, Mohamed Raouf Karray. Dynamique lacustre Holocène dans la sebkha al Kalbiyya (Tunisie centre orientale). Quaternaire, 2017, vol. 28/2, pp.239-245. 10.4000/quaternaire.8138. hal-02940778

\section{HAL Id: hal-02940778 https://hal.science/hal-02940778}

Submitted on 16 Sep 2020

HAL is a multi-disciplinary open access archive for the deposit and dissemination of scientific research documents, whether they are published or not. The documents may come from teaching and research institutions in France or abroad, or from public or private research centers.
L'archive ouverte pluridisciplinaire HAL, est destinée au dépôt et à la diffusion de documents scientifiques de niveau recherche, publiés ou non, émanant des établissements d'enseignement et de recherche français ou étrangers, des laboratoires publics ou privés. 


\section{Quaternaire}

Revue de l'Association française pour l'étude du

Quaternaire

vol. 28/2 | 2017

Volume 28 Numéro 2

\section{Dynamique lacustre Holocène dans la sebkha al Kalbiyya (Tunisie centre orientale)}

Holocene lacustrine dynamic in sebkha al Kalbiyya (eastern central Tunisia)

Abdelkarim Boujelben, Vincent Lebreton et Mohamed Raouf Karray

\section{(2) OpenEdition}

\section{Journals}

Édition électronique

URL : http://journals.openedition.org/quaternaire/8138

DOI : 10.4000 /quaternaire.8138

ISSN : 1965-0795

Éditeur

Association française pour l'étude du quaternaire

Édition imprimée

Date de publication : 29 mai 2017

Pagination : 239-245

ISSN : 1142-2904

Ce document vous est offert par Muséum national d'histoire naturelle

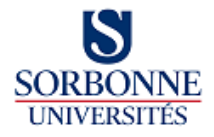

Référence électronique

Abdelkarim Boujelben, Vincent Lebreton et Mohamed Raouf Karray, « Dynamique lacustre Holocène dans la sebkha al Kalbiyya

(Tunisie centre orientale) », Quaternaire [En ligne], vol. 28/2 | 2017, mis en ligne le 01 juin 2017, consulté le 16 septembre 2020. URL : http://journals.openedition.org/quaternaire/8138 ; DOI : https:// doi.org/10.4000/quaternaire.8138 


\title{
DYNAMIQUE LACUSTRE HOLOCÈNE DANS LA SEBKHA AL KALBIYYA (TUNISIE CENTRE ORIENTALE)
}

\author{
Abdelkarim BOUJELBEN ${ }^{1,2}$, Vincent LEBRETON ${ }^{3} \&$ Mohamed Raouf KARRAY $^{1}$
}

\begin{abstract}
RÉSUMÉ
Une carotte profonde de 31,47 m a été prélevée dans la sebkha al Kalbiyya en Tunisie centre orientale. Le présent travail se focalise sur la section médiane riche en malacofaune lacustre, entre 11,18 et 16,19 m de profondeur. Les premiers résultats obtenus sur le fonctionnement, les fluctuations et les récurrences lacustres holocènes de la sebkha sont présentés. La description lithoséquentielle détaillée de la section a conduit à identifier trois grands types de faciès et d'environnements de dépôt (lacustre, palustre-lacustre et palustre-sebkha) et à définir onze phases majeures de sédimentation associées à l'alternance de trois systèmes de fonctionnement paléohydrologique de type lac pérenne, lac temporaire et lac temporaire-sebkha. Ces données, couplées à trois datations radiocarbone, ont permis de cerner deux périodes de récurrences lacustres d'amplitude différente. La première période, entre 6971 et 5596 cal BP, est de grande amplitude, quand la seconde, entre 5596 et 4970 cal BP, est de faible amplitude.
\end{abstract}

Mots-clés : al Kalbiyya, sebkha, description lithoséquentielle, datation $\mathrm{AMS}{ }^{14} \mathrm{C}$, récurrences lacustres, fonctionnement hydrologique, Holocène, Tunisie centre orientale

\section{ABSTRACT}

HOLOCENE LACUSTRINE DYNAMIC IN SEBKHA AL KALBIYYA (EASTERN CENTRAL TUNISIA)

A $31.47 \mathrm{~m}$ long continuous core has been retrieved from sebkha al Kalbiyya in eastern central Tunisia. The study focuses on the 11.18 - $16.19 \mathrm{~m}$ interval of the core, and its rich lacustrine malacological content. Based on the preliminary results, the paper aims to discuss the Holocene sebkha function as a lake, associated to fluctuations and recurrences of lacustrine deposits. The detailed lithosequential description of the section has led to identify three major types of facies and depositional environments (lacustrine, palustrine-lacustrine and palustrine-sebkha) and to define eleven major sedimentation phases associated with the alternation of three paleohydrological systems of perennial lake, temporary lake and temporary lake-sebkha types. These data coupled with three radiocarbon datings allowed to distinguish two periods of lacustrine recurrences of different amplitude. The first period, between 6971 and $5596 \mathrm{cal} \mathrm{BP}$, is of great amplitude, while the second, between 5596 and $4970 \mathrm{cal}$ BP, is of low amplitude.

Keywords: al Kalbiyya, sebkha, lithosequential description, AMS ${ }^{14} \mathrm{C}$ dating, lake recurrences, hydrological functioning, Holocene, eastern central Tunisia

\section{1 - INTRODUCTION : ÉTAT DE LA QUESTION ET CADRE DE L'ÉTUDE}

En milieu semi-aride, l'intérêt porté aux sebkhas tient dans le fait qu'elles constituent des lieux à haut potentiel d'archivage des dynamiques sédimentaires et environnementales. En ce sens, des recherches paléoenvironnementales, basées principalement sur l'étude des archives morphosédimentaires, ont été entreprises dans la sebkha al Kalbiyya, la plus grande zone humide du Sahel de Sousse en Tunisie centre orientale. Des observations de terrain (Karray, 1990a, 1993) et des investigations de subsurface avec la réalisation de treize sondages à la tarière manuelle de type Edelman (Boujelben, 2015) ont mis en évidence la présence sur les rives est et sud-est d'une falaise lacustre morte et d'un dépôt de plage à Cerastoderma glaucum, ainsi que la forte hétérogénéité et l'originalité des remplissages sédimentaires marqués par l'intercalation d'au moins quatre autres niveaux repères à Cerastoderma glaucum. Ces derniers ont fait l'objet de deux datations radiocarbone dans le but de caler les dépôts de la falaise et la plage lacustre. Les âges obtenus sont respectivement de $8052 \pm 168$ BP (Karray, 1993) et $5632 \pm 218 \mathrm{BP}$ (Boujelben, 2015). Ces données sont complétées par les résultats d'analyses sédimentologiques (Boujelben, 2015) et d'investigations archéologiques sur

\footnotetext{
${ }^{1}$ Laboratoire de Cartographie Géomorphologique des Milieux, des Environnements et des Dynamiques (CGMED, LAB-ST-04), TUNIS, Tunisie. Courriel : aboujelben_geo@yahoo.fr

${ }^{2}$ UMR 6554 CNRS, LETG

${ }^{3}$ HNHP UMR 7194 CNRS, Département Homme et Environnement, Muséum national d'Histoire naturelle, PARIS, France
} 
les bordures de la sebkha (Crema, 2006 ; Mulazzani, 2010). Le tout a permis d'établir - en relation avec la modification des signaux de forçages naturels et anthropiques - un schéma global de l'évolution des processus, des conditions et des milieux de sédimentation, des environnements et du fonctionnement hydrologique d'al Kalbiyya durant l'Holocène (Boujelben, 2015).

Parallèlement à ces travaux, une action thématique ayant pour objectif l'analyse morphosédimentaire et paléoécologique de la sebkha a été engagée entre l'UMR 7194 CNRS HNHP (département Homme et Environnement, Muséum national d'Histoire naturelle, France) et le laboratoire CGMED (LAB-ST-04, Tunisie) afin d'accroître le corpus de données multidisciplinaires, de remédier aux lacunes chronologiques et d'accéder à des dépôts plus anciens et plus profonds. Dans ce cadre, un sondage carotté profond de $31,47 \mathrm{~m}$ a été effectué et une série d'analyses multi-paramètres est engagée. Ce travail vise à présenter les résultats préliminaires de la description lithoséquentielle et des datations ${ }^{14} \mathrm{C}$ AMS des dépôts lacustres fossilifères de la partie médiane de la carotte.

\section{2 - SITE D'ÉTUDE : LA SEBKHA AL KALBIYYA}

La sebkha al Kalbiyya se situe à environ $25 \mathrm{~km}$ au nordouest de la ville de Sousse, dans le domaine sud méditerranéen semi-aride, en Tunisie centre orientale (fig. 1.1). Elle s'étend sur $102 \mathrm{~km}^{2}$, occupant la partie centrale et septentrionale d'une vaste dépression semi-endoréique. Cette dernière est directement ouverte sur les basses plaines kairouanaises au sud (50 - $60 \mathrm{~m})$ et est cernée par une topographie de croupes (Dhraa) et de collines bien marquées à l'est entre 100 et $165 \mathrm{~m}$ d'altitude (fig. 1.2). La dépression qui loge la sebkha al Kalbiyya est d'origine tectonique, faisant partie d'une large gouttière mioplio-quaternaire allongée de Kairouan jusqu'au golfe de Hammamet. Elle correspond à une cuvette synclinale subsidente, délimitée par un jeu croisé de failles et à l'aplomb d'une forte anomalie gravimétrique négative (Castany, 1948 ; Burollet, 1956 ; Lamti, 1988 ; Amari \& Bédir, 1989 ; Tagorti, 1990 ; Karray, 1990b ; Bédir, 1995).

Al Kalbiyya reçoit les apports proches de petits oueds tributaires. Elle est également alimentée par les apports allogènes des oueds Nebhana, Merguelil et Zéroud qui parcourent des centaines de kilomètres depuis les Hautes Steppes et les Basses Steppes à l'ouest jusqu'au Sahel à l'est. L'ensemble forme ainsi un bassin hydrographique grand de plus de $15000 \mathrm{~km}^{2}$, drainant toute la Tunisie centrale depuis les frontières tuniso-algériennes. La sebkha al Kalbiyya en représente le principal réceptacle hydro-sédimentaire.

À l'occasion de phénomènes pluvio-hydrologiques exceptionnels, al Kalbiyya peut être remplie jusqu'au seuil situé à $19 \mathrm{~m}$ et déborder dans l'oued al Manfas as-Sod au nord-est. Cet oued constitue un déversoir qui assure exceptionnellement l'évacuation des trop-pleins de la sebkha vers la Méditerranée par l'intermédiaire de la sebkha littorale Halq al Minjil (Karray, 1990b, 1993 ; Boujelben, 2002, 2015).

\section{3 - MATÉRIEL ET MÉTHODE D’ANALYSE}

Le sondage a été implanté dans la partie centre-est de la sebkha, à environ $700 \mathrm{~m}$ du rivage oriental actuel. La carotte SK10-C1, prélevée avec un carottier Technidrill (double type T6, diamètre $116 \mathrm{~mm}$ ) est longue de $31,47 \mathrm{~m}$. $\mathrm{Au}$ laboratoire, la colonne sédimentaire a été décrite et photographiée à haute résolution. Au niveau macroscopique, de nombreuses variations dans la couleur, la granularité et le contenu des sédiments sont observées. Le log lithostratigraphique présente quatre unités sédimentologiques majeures, témoignant de l'histoire hydro-morphosédimentaire de la sebkha (fig. 1.3). De la base du carottage jusqu'à 28,45 m de profondeur, l'unité A est dominée par des sédiments argilo-limoneux compacts contenants des nodules et des concrétions calcaires millimétriques. $\mathrm{Ce}$ faciès sédimentaire est bien reconnu sur les rives de la sebkha (Karray, 1990a, 1993), mais il est aussi caractéristique et fréquemment identifié en Tunisie centrale où il est attribué au Pléistocène supérieur (Boujarra \& Gammar, 1993 ; Karray, 1993 ; Boujelben, 2002, 2015 ; Brahim, 2005 ; Ben Jmaa, 2008 ; Bkhairi, 2013). L'unité B, entre $28,45 \mathrm{~m}$ et $16,19 \mathrm{~m}$ de profondeur, est formée par un dépôt compact et hétérogène, composé de limons sableux et argileux, de sables fins et d'argiles limoneuses, avec des horizons fins d'oxyde de fer et de gypse et des amas calcaires de taille millimétrique. L'unité C, entre $16,19 \mathrm{~m}$ et $11,18 \mathrm{~m}$, est constitué d'un remplissage limono-argileux, sableux et limoneux, intercalé de niveaux à limnées et à Cerastoderma glaucum. À partir de 11,18 m jusqu'à la surface, l'unité D présente une sédimentation fine d'argiles et de limons, souvent oxydée et avec des lits discontinus de gypse. Elle montre au milieu, l'intercalation d'un niveau de $5 \mathrm{~cm}$ d'épaisseur riche en limnées. L'ensemble du carottage SK10-C1 enregistre ainsi la transition Pléistocène supérieur-Holocène et tout l'Holocène.

Pour ce travail, l'intérêt est porté sur l'unité $\mathrm{C}$ dans la partie médiane de la carotte SK10-C1, au vu de son contenu malacologique. Les Cerastoderma glaucum sont des bivalves tolérants aux conditions euryhalines et eurythermiques qui règnent dans les biotopes lacustres et lagunaires (Rosso, 1979). Ils représentent avec les limnées, gastéropodes d'eau douce, d'importants bioindicateurs environnementaux permettant de reconstituer le régime et le fonctionnement paléohydrologique de la sebkha, tout en se prêtant à la datation.

La description fine du matériel sédimentaire a permis d'établir une colonne stratigraphique décrivant dans le détail la superposition des séquences sédimentaires d'épaisseur variable, centimétrique à décimétrique. Les séquences répétitives et génétiquement liées traduisent des mêmes provenances, dynamiques et environnements sédimentaires. Elles sont groupées en trois grandes catégories de faciès : lacustre, palustre-lacustre et palustresebkha. Chaque faciès explique une phase majeure de 

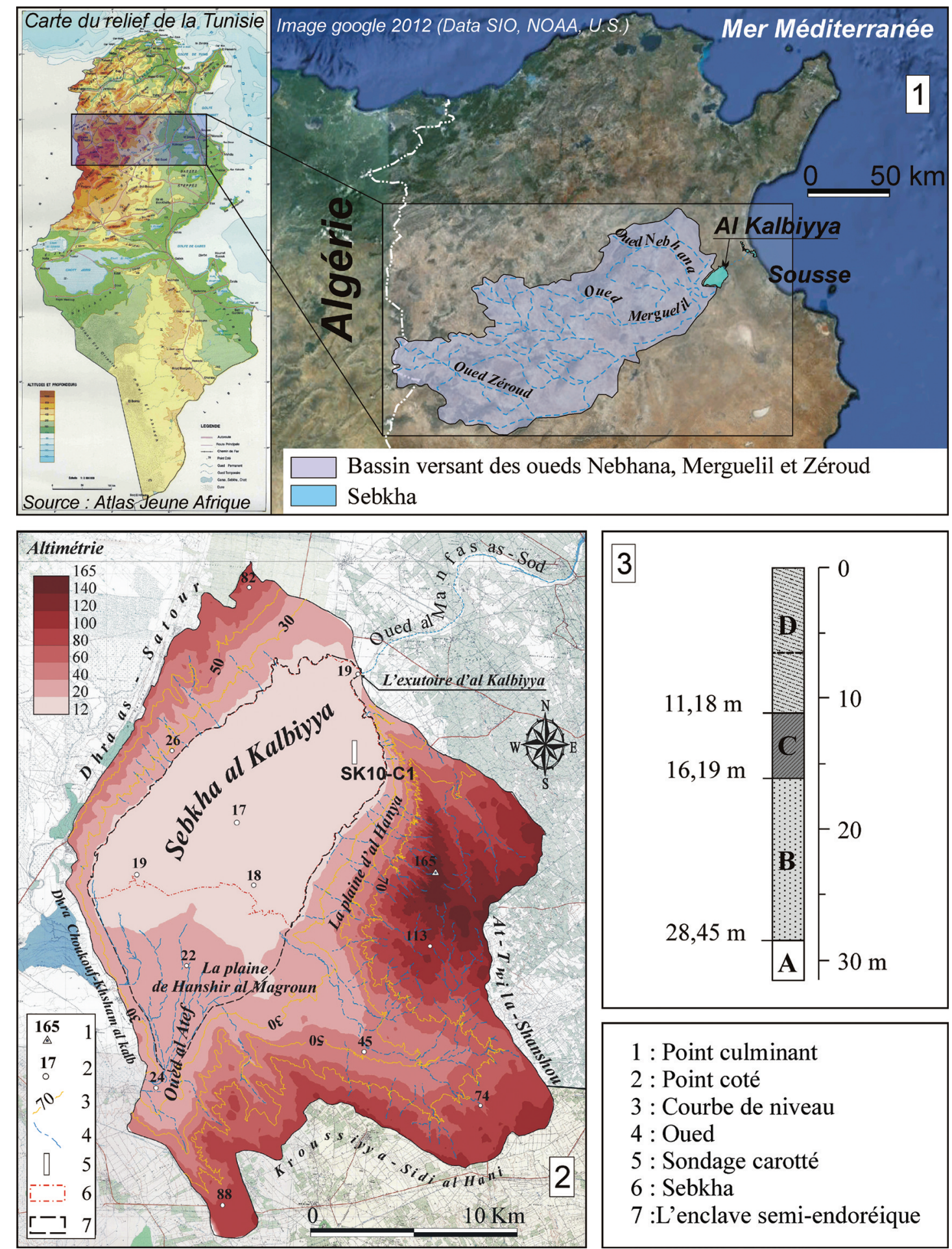

\section{$1:$ Point culminant}

2 : Point coté

3 : Courbe de niveau

4 : Oued

5 : Sondage carotté

6 : Sebkha

7 :L'enclave semi-endoréique

Fig. 1 : (1) Sebkha al Kalbiyya dans son contexte régional ; (2) Cadre orohydrographique de la sebkha al Kalbiyya et site d'implantation du sondage carotté ; (3) Unités sédimentaires principales de la carotte SK10 C1.

A- argiles limoneuses compactes avec nodules et concrétions calcaires ; B- limons, sables et argiles compacts avec horizons d'oxyde de fer et de gypse et amas calcaires ; C- limons argileux et sables limoneux à limnées et Cerastoderma glaucum; D- argiles et limons, souvent oxydés avec lits discontinus de gypse et un niveau à limnées (profondeur 6,40 - 6,45 m, épaisseur $5 \mathrm{~cm}$ ).

Fig. 1: (1) Sebkha al-Kalbiyya in its regional context; (2) Orohydrographic frame of Sebkha al Kalbiyya and location of core SK10-C1; (3) Main sedimentary units of core SK10-C1: A-compact silt clays with nodules and calcareous concretions; B-silt, sand and clay compacts with iron oxide and gypsum horizons and calcareous clusters; $C$ - clay loams and silty sands with limnes and Cerastoderma glaucum; D-Clays and silts, often oxidized, with discontinuous gypsum beds, and a limne layer (depth: 6.40 - $6.45 \mathrm{~m}$, thickness $5 \mathrm{~cm}$ ).

sédimentation et d'installation d'un milieu de dépôt et d'un système particulier de fonctionnement hydrologique. Les limites stratigraphiques entre ces faciès sont matérialisées par l'apparition ou la disparition des biomarqueurs ou par un changement drastique dans les remplissages.
Trois datations ${ }^{14} \mathrm{C}$ AMS ont été effectuées sur des fragments et des valves de Cerastoderma glaucum (tab. 1). Les âges ${ }^{14} \mathrm{C}$ BP ont été convertis en âges calibrés BP et $\mathrm{BC}$ à l'aide du logiciel Calib Rev 7.0.4 (Stuiver \& Reimer, 1993). La valeur de correction du $\delta \mathrm{R} 58 \pm 85$ a été utilisée (Reimer \& Mc 


\begin{tabular}{|c|c|c|c|c|c|}
\hline $\begin{array}{l}\text { Référence } \\
\text { Beta }\end{array}$ & Matériel daté & $\begin{array}{l}\text { Profondeur } \\
\text { (m) }\end{array}$ & $\underset{B P}{\hat{A} g e}{ }^{14} C$ & $\begin{array}{c}\text { Âge calibré à } 2 \sigma \\
(\text { cal. BP })\end{array}$ & $\begin{array}{l}\text { Âge calibré à } 2 \sigma \\
\text { (cal. BC) }\end{array}$ \\
\hline Beta-290407 & Cerastoderma glaucum & 11,4 & $4770 \pm 40$ & $4771(\mathbf{4 9 7 0}) 5271$ & $3322(\mathbf{3 0 2 0}) 2822$ \\
\hline Beta-290408 & Cerastoderma glaucum & 12,75 & $5290 \pm 40$ & $5360(\mathbf{5 5 9 6}) 5845$ & $3896(\mathbf{3 6 4 6}) 3411$ \\
\hline Beta-290409 & Cerastoderma glaucum & 13,8 & $6530 \pm 40$ & 6735 (6971) 7193 & $5244(\mathbf{5 0 2 1}) 4786$ \\
\hline
\end{tabular}

Tab. 1 : Datations AMS ${ }^{14} \mathrm{C}$ des remplissages lacustres de la sebkha al Kalbiyya.

Âges calibrés avec borne minimale à 2 sigmas, âge médian, borne maximale à 2 sigmas.

Tab. 1: AMS ${ }^{14} \mathrm{C}$ dates of the lacustrine deposits of sebkha al Kalbiyya (calibrated ages with 2 sigmas range minimum, median age, 2 sigmas range maximum).

Cormac, 2002). En l'absence de référentiel sur l'effet réservoir local, cette même valeur avait été adoptée par Saliège et al. (2013) pour calibrer les coquilles de Cerastoderma glaucum provenant de la sebkha littorale Halq al Minjil, à $25 \mathrm{~km}$ à l'aval de la sebkha al Kalbiyya. Un modèle âge-profondeur et des âges médians cal BP ont été calculés en utilisant le même logiciel.

\section{4- RÉSULTATS}

\section{1 - STRATIGRAPHIE SÉQUENTIELLE ET FACIÈS SÉDIMENTAIRES}

L'unité C de la carotte SK10-C1 montre la succession rapide de 71 séquences. La séquence basale est épaisse de 1,19 m, mais les 70 autres font chacune entre 1 et $15 \mathrm{~cm}$ (fig. 2). Ces séquences sont hétérogènes et intercalées d'horizons hydromorphes, organiques et/ou évaporatiques, avec présence ou absence de restes malacologiques. Trois faciès sédimentaires ont été identifiés (fig. 2). Le faciès 1 est lacustre. Il contient quelques petits fragments de nacre, des limnées et des débris ainsi que des valves parfois connectées de Cerastoderma glaucum de tailles variables. Ce faciès 1 regroupe 55 séquences, épaisses de $423 \mathrm{~cm}$ au total. La texture est sableuse, limoneuse et limono-argileuse et la couleur est gris, gris foncé et gris olive. Le sédiment est piqueté de grains noirs. Il renferme des taches diffuses et des horizons fins noirs et gris olive et parfois des lits millimétriques de gypse sous forme de disques et lentilles. Le faciès 2 est palustre-lacustre, avec huit séquences d'une épaisseur totale de $44,5 \mathrm{~cm}$. Il ne contient pas de coquilles. Le matériel, constitué de sables et de limons argileux lités, présente les mêmes caractéristiques que le faciès 1 . Le faciès 3 est un faciès de transition palustre-sebkha. Il regroupe huit séquences d'une épaisseur totale de $33 \mathrm{~cm}$ (6,5\% de la section sédimentaire). Le dépôt est plus fin, composé de limons et de limons argileux de couleurs variées (vert olive, gris olive, gris, gris foncé). Il se distingue par une forte concentration du gypse dans le sédiment, sous forme de lits de disques et de macrocristaux lenticulaires. Il montre également l'intercalation de taches et d'horizons fins d'oxydation de fer et des gravillons limoneux roulés et oxydés de 2 à $3 \mathrm{~mm}$ de diamètre. Parfois, des grains et des lits millimétriques discontinus noirs et gris olive s'intercalent dans les sédiments.

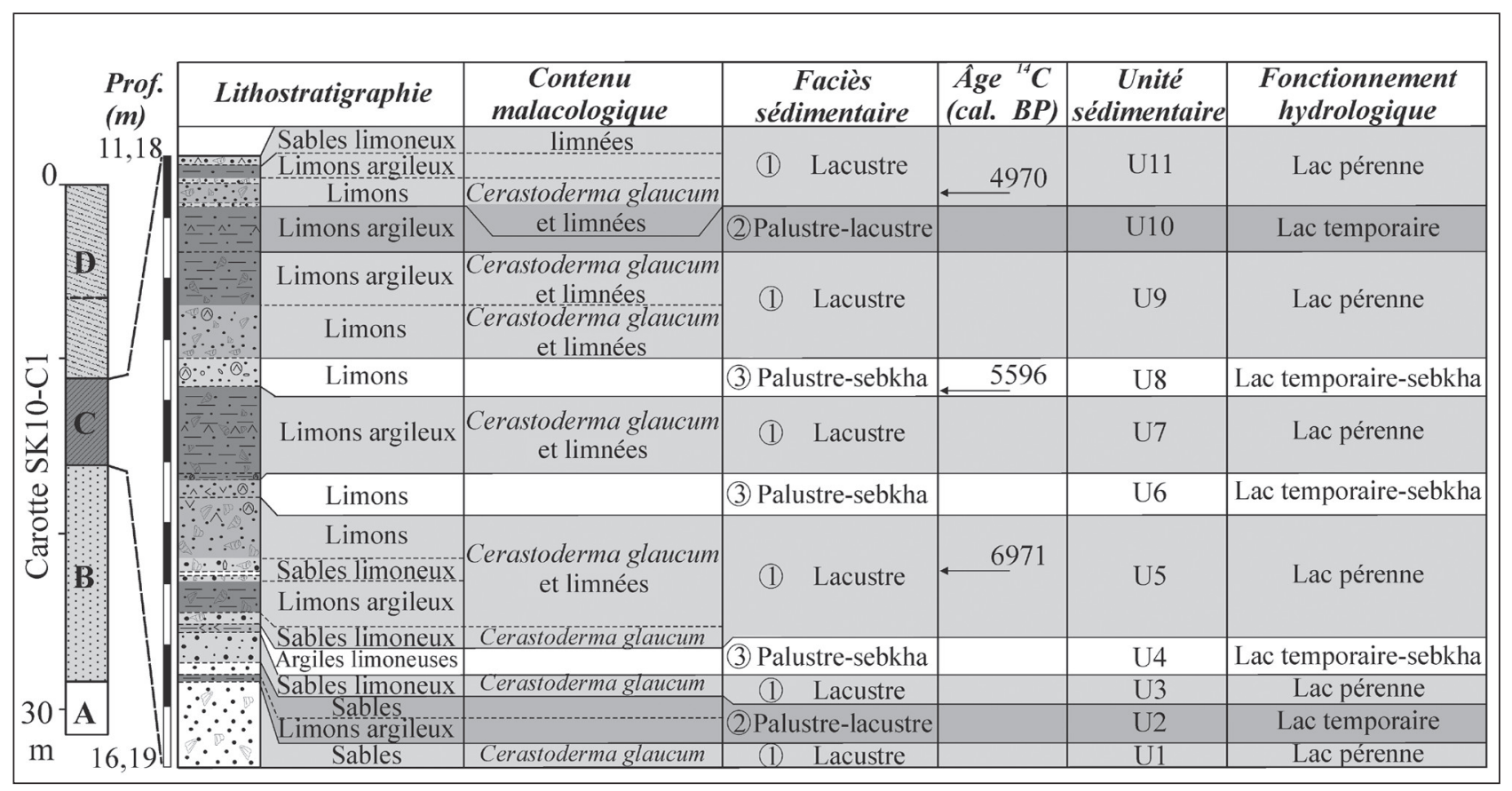

Fig. 2 : Dépôts lacustres holocènes (unité $\mathrm{C}$ ) d'al Kalbiyya : stratigraphie séquentielle, faciès sédimentaires et milieux de sédimentation. Fig. 2: Holocene lacustrine deposits (Unit C) of al Kalbiyya: sequential stratigraphy, sedimentary facies and sedimentation environments. 


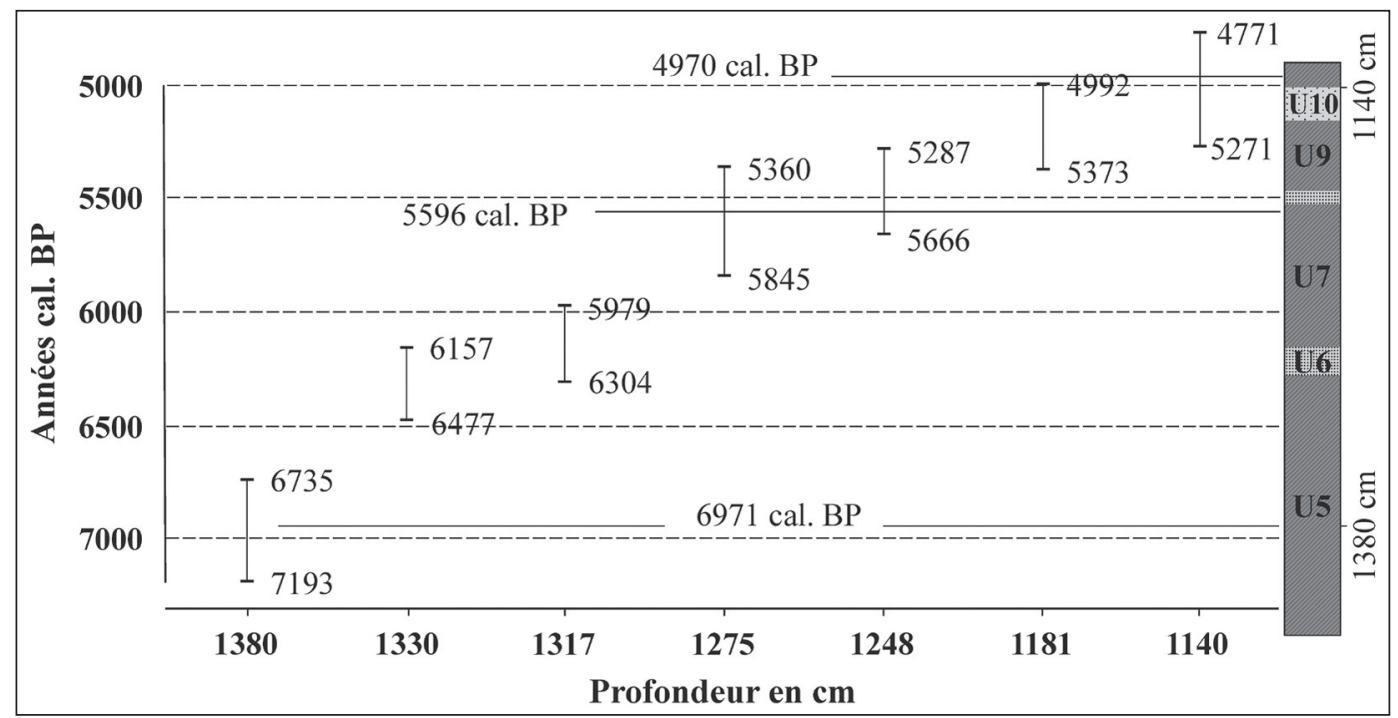

Fig. 3 : Modèle âge-profondeur de la section sédimentaire entre 11,4 et $13,8 \mathrm{~m}$ de profondeur. fig. 3: Age-depth model of the sedimentary section between 11.4 and $13.8 \mathrm{~m}$ depth.

\section{2 - CHRONOLOGIE}

Seule la moitié supérieure de la section sédimentaire étudiée est datée à ce jour par son contenu malacologique. Trois datations AMS ${ }^{14} \mathrm{C}$ ont été réalisées sur l'espèce Cerastoderma glaucum, prélevée sur trois niveaux entre 11,4 et $13,8 \mathrm{~m}$ de profondeur (tab. 1). Un modèle âge-profondeur et des âges médians cal BP ont été calculés (fig. 3). Ce modèle d'âge préliminaire est toutefois sujet à des variations du stockage sédimentaire et des discontinuités sédimentaires ont pu survenir entre ces trois dates obtenues, notamment lors de périodes arides marquées par l'assèchement de la sebkha et l'érosion du fond de la dépression. Il reste toutefois possible d'apprécier l'ordre de grandeur du taux de sédimentation pour le réceptacle d'al Kalbiyya. L'accumulation, épaisse de 2,4 m, est mise en place entre 6971 et 4970 cal BP à l'Holocène moyen. Les rythmes d'accumulation varient alors entre 0,7 et $2 \mathrm{~mm} / \mathrm{an}$ respectivement pour les périodes 6971 - $5596 \mathrm{cal} \mathrm{BP}$ et 5596 - $4970 \mathrm{cal} \mathrm{BP}$. Au vu du modèle âge-profondeur, un taux de remplissage moyen de 1,6 mm/an est calculé. Ce taux, relativement lent, témoigne des faibles dynamiques de remblaiement du fond de la sebkha al Kalbiyya. A titre indicatif et en contexte de sebkha submersible, Karray (1993) a avancé deux moyennes annuelles d'exhaussement du fond d'al Kalbiyya de 2,8 et $4,2 \mathrm{~cm} /$ an pour les périodes 1952 1969 et 1969-1979 en rapport avec une augmentation sensible de l'alluvionnement due aux grandes crues.

\section{5 - DISCUSSION}

\section{1 - PHASES SÉDIMENTAIRES ET SYSTĖMES DE FONCTIONNEMENT HYDROLOGIQUE}

L'organisation stratigraphique des trois faciès sédimentaires retrace la dynamique des milieux au cours de l'Holocène moyen. Elle conduit à définir onze phases majeures de sédimentation (U1-U11 ; fig. 2), associées à l'alternance de trois grands types d'environnements de dépôt et de fonctionnement paléohydrologique. La sebkha al Kalbiyya a évolué alternativement en systèmes de lacs pérennes, lacs temporaires et lacs temporaires-sebkhas.

Le faciès 1 , contenant des limnées et Cerastoderma glaucum, est typique d'un milieu de sédimentation lacustre. Les sédiments, principalement terrigènes, sont mis en place en périodes de crues dans un lac pérenne d'eau douce à saumâtre, peu profond et à forte production biocénotique. Le gypse peut être remanié et/ou de néoformation, traduisant le retrait des eaux lacustres et/ou l'évaporation de la tranche d'eau dans la cuvette lacustre. Al Kalbiyya a connu six épisodes lacustres permanents, d'ampleur variable (U1, U3, U5, U7, U9 et U11). La phase lacustre débute par une séquence sédimentaire homogène, très épaisse $(1,19 \mathrm{~m})$ et à texture sableuse traduisant des dynamiques actives de comblement du fond lacustre.

Le faciès 2, dépourvu de restes malacologiques, marque des changements dans les conditions et le fonctionnement hydrologique d'al Kalbiyya. Il est caractéristique d'environnement sédimentaire palustre-lacustre, temporairement émergé. Les deux phases de sédimentation U2 et U10 montrent ainsi l'installation d'un système de lac temporaire, très fluctuant, d'étendue variable et de profondeur généralement faible. Un tel milieu est vraisemblablement caractérisé par un court stationnement des conditions limniques expliquant l'arrêt de la production biocénotique.

L'intercalation des taches et des horizons d'oxyde de fer rouille d'une part et la forte concentration du gypse dans les sédiments du faciès 3 d'autre part, sont les témoignages d'épisodes successifs d'exondation et d'assèchement prononcés et/ou prolongés du lac sous déficit hydrique, avec possiblement des remontées périodiques de la nappe. Sous de telles conditions, al Kalbiyya évolue alors entre un milieu lacustre temporaire et un milieu de 
sebkha. Les gravillons limoneux roulés peuvent être des agrégats éolisés, mis en mouvement par voie éolienne ou des gravillons détachés et mis en roulement par l'eau.

\section{2 - DEUX PÉRIODES DE RÉCURRENCES LACUSTRES D'AMPLITUDE DIFFÉRENTE À L'HOLOCÈNE MOYEN}

La confrontation des données de la stratigraphie séquentielle avec les datations AMS ${ }^{14} \mathrm{C}$ de la section sédimentaire entre 11,4 à 13,8 $\mathrm{m}$ de profondeur, permet de distinguer, en rapport avec les fluctuations lacustres, deux périodes de récurrences lacustres d'amplitude différente.

Une première période, entre 6971 et $5596 \mathrm{cal} \mathrm{BP}$, est marquée par des récurrences lacustres de grande amplitude, avec trois phases majeures de sédimentation (U5-U7), responsables de l'exhaussement de son fond de $105 \mathrm{~cm}$ environ (au niveau du carottage). Pendant cette période, al Kalbiyya enregistre l'installation de deux épisodes lacustres pérennes (U5 et U7) séparés par un épisode de lac temporaire-sebkha (U6). Les récurrences lacustres étaient de grande amplitude. Malgré l'accroissement des apports détritiques en rapport avec une érosion fluviale dans les bassins et l'importance de la morphodynamique, les conditions environnementales devaient être plus ou moins stables.

Une seconde période, entre 5596 et 4970 cal BP, est marquée par des récurrences lacustres rapides de faible amplitude. Al Kalbiyya a fonctionné successivement en systèmes de lac temporaire-sebkha, de lac pérenne, de lac temporaire puis à nouveau de lac pérenne. La sédimentation est relativement plus dilatée, atteignant $135 \mathrm{~cm}$ avec des sédiments lacustres fossilifères dominants $(85,5 \mathrm{~cm})$. Une plus forte dynamique de comblement a caractérisé les lacs pérennes. Ainsi, la succession rapide et la courte durée du stationnement de ces systèmes, couplées à la forte hétérogénéité du matériel sédimentaire, témoignent-elles d'une instabilité de l'hydrodynamique et des environnements.

\section{3 - DES FLUCTUATIONS ET DES RÉCURRENCES PALÉOLACUSTRES D'AMPLITUDE DIFFÉRENTE SOUS CONTRÔLE CLIMATIQUE?}

Les résultats obtenus sur le fonctionnement, les fluctuations et les récurrences lacustres d'al Kalbiyya fournissent les premiers éléments d'interprétation paléoenvironnementale. Les épisodes lacustres permanents expliquent des bilans hydrologiques positifs dont la durée suffisamment longue aurait permis la prolifération des coquilles lacustres. Ces lacs pérennes résulteraient soit de périodes de maximum d'ennoyages récurrents et rapprochés (Karray, 1990a), soit d'une tendance à l'humidification du climat garantissant ainsi des régimes et des apports hydriques moins irréguliers. Les stades de lacs temporaires expriment par contre la diminution des apports hydriques dans la cuvette lacustre et la plus grande irrégularité du régime hydrologique et des écoulements qui sont de plus en plus sensibles aux fluctuations mineures du climat. Le stationnement épisodique de conditions limniques devait alors être tributaire d'événements hydro-pluviométriques extrêmes ou de succession de plusieurs années humides. Le niveau d'eau pouvait baisser et se rétracter sous l'effet combiné de la réduction ou de l'arrêt des apports hydriques et de l'évaporation. Un lac temporaire se transformait alors graduellement en sebkha exondée et salée. Un tel passage fonctionne au gré des événements hydro-pluviométriques interannuels et saisonniers.

La période entre 5596 et 4970 cal BP est marquée par des récurrences lacustres de faible amplitude qui témoignent d'une instabilité environnementale sous la contrainte d'événements climatiques rapides. Un changement paléoenvironnemental majeur survient dans al Kalbiyya autour de $4970 \mathrm{cal}$ BP à la transition entre l'Holocène moyen et supérieur. Cette date survient peu après la fin de l'African Humid Period vers 5500 cal BP où l'aridité croissante se met en place sur tout le continent nord africain (Bard, 2013). Depuis cette date, al Kalbiyya n'a plus fonctionné en système de lac pérenne, propice à la production biocénotique. Néanmoins, entre 6,45 et 6,40 m de profondeur, la carotte SK10C1 montre des limons argileux, riches en limnées. Cette phase pourrait pointer un bref nouvel épisode lacustre non daté, qui dénoterait un changement des environnements à la faveur d'un événement climatique rapide, avec reprise de la production biocénotique dans al Kalbiyya.

\section{6 - CONCLUSION ET PERSPECTIVES}

Ce travail présente les résultats préliminaires de la description lithoséquentielle haute résolution et des datations ASM ${ }^{14} \mathrm{C}$ de la phase de remplissages lacustres holocènes de la sebkha al Kalbiyya. Il met en évidence l'importance de la sédimentation lacustre fossilifère dans le remplissage total de la sebkha. Les faciès, les unités et les environnements sédimentaires identifiés permettent de reconstituer le fonctionnement du plus grand réceptacle sédimentaire de la Tunisie centrale. Avant $6971 \mathrm{cal} \mathrm{BP}$ et jusqu'à 4970 cal BP, l'histoire paléohydro-sédimentaire d'al Kalbiyya s'est déclinée en au moins onze étapes. La sebkha actuelle a connu de nombreuses fluctuations lacustres et a évolué principalement en systèmes de lacs pérennes productifs. Les périodes de récurrences lacustres étaient d'amplitudes différentes.

La poursuite des recherches, dans le cadre du projet Paléomex TransMaghreb, va porter sur l'analyse complète du remplissage holocène de la sebkha al Kelbiyya (analyses sédimentologiques, micromorphologiques, géochimiques, palynologiques, signal incendie, malacologie, datations) afin d'établir une séquence de référence pour l'Holocène du Maghreb oriental.

\section{RÉFÉRENCES BIBLIOGRAPHIQUES}

AMARI A. \& BÉDIR M., 1989 - Les bassins quaternaires du Sahel central de la Tunisie. Genèse et évolution des sabkhas en contexte décrochant compressif et distensif. Géodynamique, 4 (1), 49-65. 
BARD E., 2013 - Out of the African Humid Period. Science, 342 (6160), 808-809.

BÉDIR M., 1995 - Mécanismes géodynamiques des bassins associés aux couloirs de coulissements de la marge atlasique de la Tunisie. Thèse d'Etat, Université de Tunis, $416 \mathrm{p}$.

BEN JMAA H., 2008 - Le système endoréique de Sidi al Hani, Cherita et Mechertate : paléoenvironnements et dynamique récente. Thèse Doctorat, Université de Tunis, $251 \mathrm{p}$.

BKHAIRI A., 2013 - Le bassin versant de l'Oued Hatab (Tunisie centrale) : traits géomorphologiques, paléoenvironnement et morphodynamique. Thèse d'université, Université de Tunis, 406 p.

BOUJARRA A \& GAMMAR A., 1993 - Contribution à l'étude géomorphologique des niveaux à croûtes dans les environs de Sousse. Revue Tunisienne de Géographie, 23-24, 49-67.

BOUJELBEN A., 2002 - Le bassin de l'Oued al Manfas as-Sod et ses abords côtiers : aspects morphologiques et évolution des paysages de l'Holocène à nos jours. Mémoire de D.E.A., Faculté des Sciences Humaines et Sociales de Tunis I, 117 p.

BOUJELBEN A., 2015 - L'hydrosystème des sebkhas al Kalbiyya et Halq al Minjil (Tunisie centre orientale) : dynamiques hydromorphosédimentaires, paléoenvironnements et évolution des paysages pendant l'Holocène. Thèse de Doctorat, Universités de Tunis et de Caen Normandie, $347 \mathrm{p}$.

BRAHIM F., 2005 - Le Sahel central et méridional (Tunisie orientale) : géomorphologie et dynamique récente du milieu naturel. Faculté des lettres et des sciences humaines de Sousse, Finzi, 302 p.

BUROLLET P. F., 1956 - Contribution à l'étude stratigraphique de la Tunisie centrale. Annales des Mines et de la Géologie, 18, 345 p.

CASTANY G., 1948 - Les fossés d'effondrement de Tunisie : géologie et hydrologie. $1^{\text {er }}$ fascicule: Plaine de Grombalia et cuvettes de la Tunisie orientale. Annales des mines et de la géologie, Tunis, $126 \mathrm{p}$.

CREMA E.R., 2006 - Campagna di survey 2006 per la missione ad hergla, Tunisia, Relazione preliminare. Rapport inédit de fin de mission, Projet de recherche tuniso-italien pour l'étude de la préhistoire de Hirgla, 2003-2007, INP-IsIAO, 16 p.

KARRAY M. R., 1990a - Un dépôt lacustre à cardium d'âge holocène dans la sabkha Kelbia (Tunisie orientale). Notes du Service Géologique, Tunisie, 56, 85-90.
KARRAY M. R., 1990b - L'évolution du fond d'el Kelbia depuis 1892. Revue Tunisienne de Géographie, 19, 167-180.

KARRAY M. R., 1993 - Sebkhet el Kelbia (Tunisie orientale) : dynamique récente et changements actuels 1892-1992. Actes de $2^{\mathrm{e}}$ Congrès des Géographes Africains, Rabat et Agadir 19-24 avril 1993, 141-166.

LAMTI L., 1988 - Analyse morphostructurale par techniques cartographiques et télédétection spatiale sous SIG : application à la Sebkha el Kelbia et ses environs. Mémoire de D.E.A., Faculté des Sciences de Tunis, Université de Tunis, 70 p.

MULAZZANI S., 2010 - L'habitat épipaléolithique de SHM-1 et les sites environnants au bord de la sebkha-lagune de Halk el Menjel (Hergla-Tunisie) entre le VII et le VIe millénaire Cal BC. Thèse de Doctorat, Université Paris 1, Vol. I, 568 p.

REIMER E P. J. \& MCCORMAC F. G, 2002 - Marine radiocarbon reservoir corrections for the Mediterranean and Aegean seas. Radiocarbon, 44 (1), 159-166.

ROSSO J.C., 1979 - Etude des organismes A - Mollusques testacés (Macrofaune). In Burollet P. F., Clairefond P. et Winnock E. (Ed), La mer Pélagienne. Géologie Méditerranéenne, 6 (1), 141-170.

SALIÈGE J.-F., MAGNANI G. \& MULAZZANI S., 2013 - Datations ${ }^{14} \mathrm{C}$ de la rammadiya SHM-1, Ouvrage collectif, In Mulazzani S. (éd.), Le Capsien de Hergla (Tunisie) - Culture, environnement et économie. Reports in African Archaeology, 4, Africa Magna Verlag, Frankfurt, 145-150.

STUIVER M. \& REIMER P.J., 1993 - Extended 14C data base and revised $C A L I B 3.0{ }^{14} \mathrm{C}$ Age calibration program, Radiocarbon, 35 (1), 215-230.

TAGORTI M. A., 1990 - Dynamique de couverture, genèse et évolution du complexe lagunaire Sebkha Halk el Mengel-Sabkha Assa el Jeriba (Sahel de Sousse) : analyse séismostratigraphique, géochimie et échanges hydrologiques saisonniers. Thèse de Doctorat, Faculte des Sciences de Tunis, Université de Tunis, 162 p. 